\title{
ANTIDEPRESSANT-LIKE EFFECTS OF THE AMPA POTENTIATOR S47445 ARE ASSOCIATED WITH CHANGES IN HIPPOCAMPAL BDNF AND mTOR SIGNALING IN BULBECTOMY MICE MODEL
}

P.2.a.011

\author{
F. Pilar-Cuellar ${ }^{1,2}$, E. Castro ${ }^{1,2}$, S. Bretin ${ }^{3}$, E. Mocaer ${ }^{3}$, A. Pazos ${ }^{1,2}$ and A. Diaz ${ }^{1,2}$
}

1 Instituto de Biomedicina y Biotecnología de Cantabria IBBTEC, Department of Molecular and Cellular Signalling, Santander, Spain.

2 CIBERSAM- Centro de Investigación Biomédica en Red de Salud Mental- Instituto de Salud Carlos III, Department of Molecular and Cellular Signalling, Santander, Spain.

3 Institut de Recherches Internationales Servier, Pôle d'Innovation Thérapeutique Neuropsychiatrie, Suresnes Cedex, France.

\section{Purpose of the study}

The positive allosteric modulator of glutamate AMPA-type receptors $\$ 47445$ increases neurotrophic levels, enhancing synaptic plasticity and procognitive functions in aged rodent models [1-4]. Moreover, the chronic administration of this compound induces antidepressant- and anxiolytic-like effects in a wide variety of animal models of depression [5]. Here, we have assessed the behavioral effects on depression and anxiety paradigms after the chronic administration of 547445 in the bilateral olfactory bulbectomy (OBX) mice model of depression. In addition, we have evaluated the changes in neurotrophic (BDNF) and neuroplastic markers (mTOR pathway) in the hippocampus, which are associated to the antidepressant effect.

\section{Methods}

Animals: Adult C57/BL6J male mice were submitted to OBX and received 547445 at 3 doses $(1-3-10 \mathrm{mg} / \mathrm{kg} / \mathrm{day}$, ip.) or fluoxetine (18 mg/kg/day, i.p.) for 4 weeks ( $n=15 /$ group). OBX-mice were behaviorally assessed following 3 and 4 weeks of treatment.

Open-field test (locomotor activity): Open-field was performed under high luminance conditions (400 Ix), and the

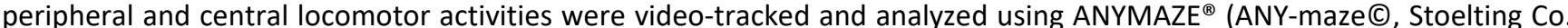
UK)

Western Blot: At the end of behavioral testing (day 28), and after a $24 \mathrm{~h}$ washout period, mice were sacrificed and the hippocampi were dissected. Whole protein homogenates were extracted using Tripure ${ }^{\mathrm{TM}}$ Isolation Reagent (Sigma-Aldrich) according to the manufacturer's instructions. Protein quantification was performed according to the Lowry method (Lowry et al., 1951). About 50-60 $\mu \mathrm{g}$ of protein were resolved on 8.5\% SDS-PAGE and transferred to PVDF or nitrocellulose membranes. Membranes were incubated in rabbit anti-BDNF (Santa Cruz Biotechnology), mTOR (Sigma-Aldrich), phosphomTOR, 4EBP1 and phospho-4EBP1 (Cell Signalling). After washing in TBS-T (TBS/0.05\% Tween 20), membranes were incubated with horseradish peroxidase conjugated anti-mouse secondary antibody. Secondary antibodies were detected with ECL Advance kit (GE Healthcare Europe GmbH, Munich, Germany). Blot quantifiquations were performed by densitometric scanning using Scion Image Software. The densitometry values were normalized with respect to the values obtained with anti- $\beta$-tubulin antibody (Sigma-Aldrich). Data are expressed as a percentage of the WT group values (100\%) and are presented as the mean \pm S.E.M.

Data analysis: Statistical analysis was made using an unpaired Student's $t$-test, and one-way ANOVA test. The significance level was set at $p<0.05$

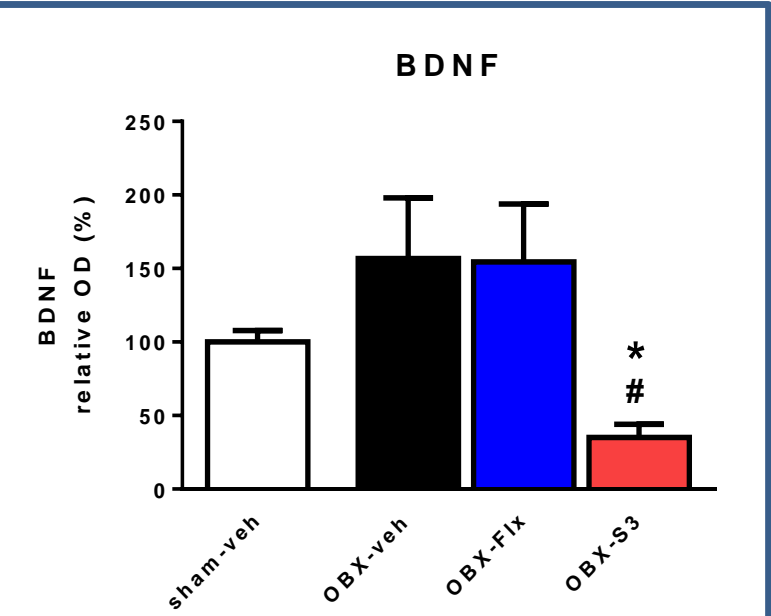

Figure 3. BDNF. A trend to increased BDNF was observed in OBX model. This was reverted after 28 days of treatment with $\mathrm{S} 47445 \quad 3 \mathrm{mg} / \mathrm{kg} /$ day. No effect was observed with fluoxetine $18 \mathrm{mg} / \mathrm{kg} /$ day. One-way ANOVA, ${ }^{*} p<0.05$ vs OBX-veh, \#p< 0.05 vs OBX-FlX.

\section{Results}

A
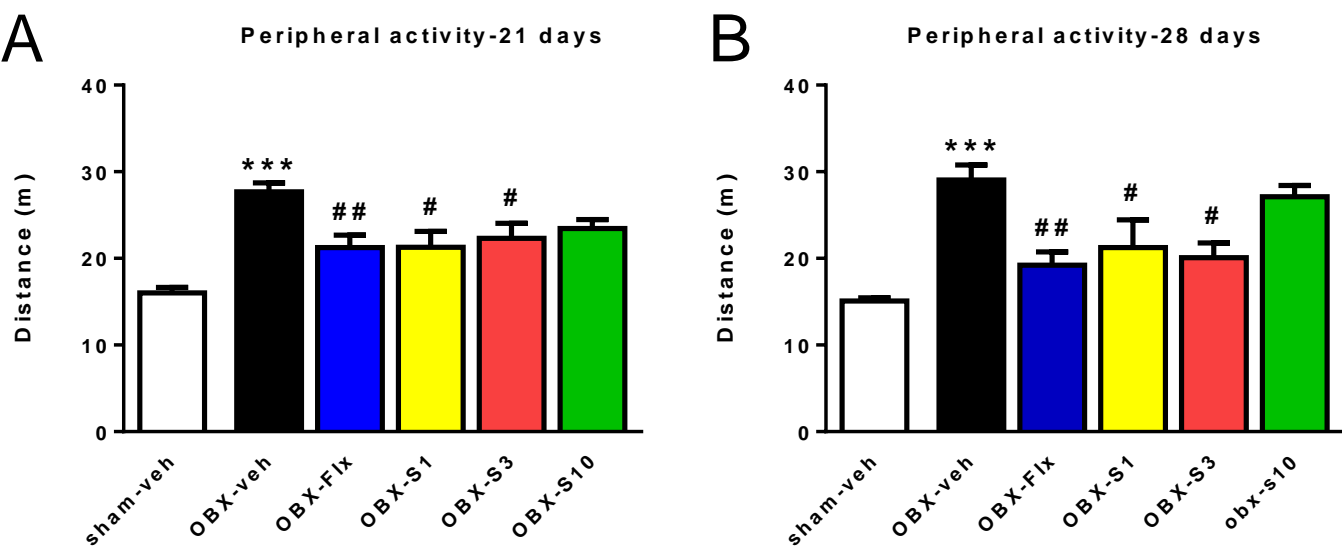

Figure 1. Open field test. Reversion of OBX-induced hyperactivity after 21 and 28 days of treatment with S47445 at 1 and $3 \mathrm{mg} / \mathrm{kg} / \mathrm{day}$, i.p. The effect was similar to that induced by fluoxetine $18 \mathrm{mg} / \mathrm{kg} / \mathrm{day}$.

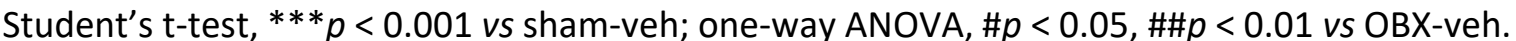

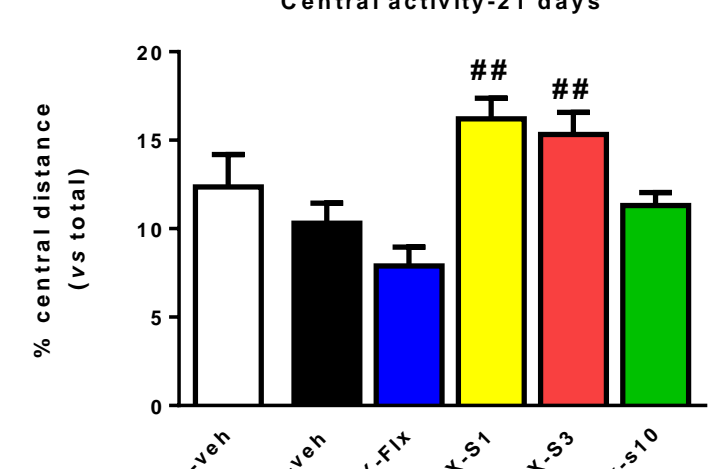

Figure 2. Open field test. Anxiolytic-like effect in the OBX model after 21 days of treatment with $\$ 47445$ at 1 and $3 \mathrm{mg} / \mathrm{kg} /$ day. No effect was observed after chronic fluoxetine treatment $(18 \mathrm{mg} / \mathrm{kg} / \mathrm{day})$. Oneway ANOVA, \#\#p $<0.01$ vs OBX-veh.

\section{CONCLUSION}

Chronic administration of $\$ 47445$ induced an antidepressant-like effect in the OBX mice model at low-medium doses (1 and 3 $\mathrm{mg} / \mathrm{kg} /$ day).

S47445 counteracts OBX-induced trend of changes by decreasing BDNF and mTOR activation, and by increasing p4EBP1 expression in the hippocampus. Fluoxetine had no effect on these parameters.

Thus, the antidepressant effects of $\$ 47445$ were associated with the modulation of hippocampal BDNF/mTOR/p-4EBP1 signalingpathway in the OBX mice model of depression, in contrast to that observed with fluoxetine.

A

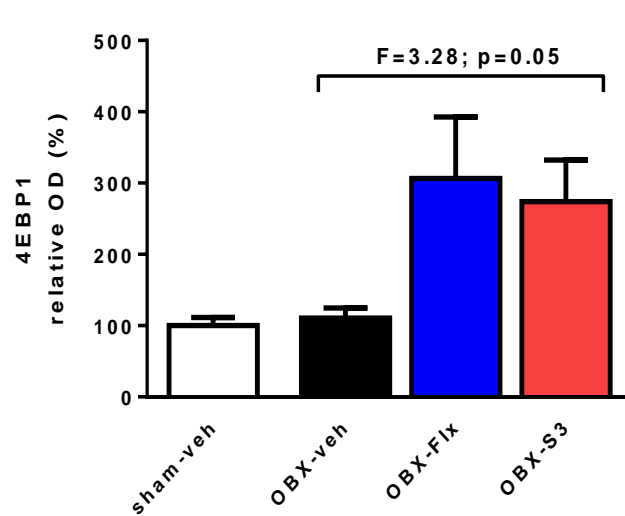

Figure 5. 4EBP1 (A), p-4EBP1 (B) and ratio $p$-4EBP1/4EBP1 (C). Increased expression of $4 \mathrm{EBP} 1$ with $\mathrm{S47445}$ at 3 $\mathrm{mg} / \mathrm{kg} / \mathrm{day}$ and fluoxetine at 18 $\mathrm{mg} / \mathrm{kg} /$ day (A). Increased expression of the phosphorylated form after 28 days of treatment with $\$ 47445$ at 3 $\mathrm{mg} / \mathrm{kg} /$ day (B). One-way ANOVA, ${ }^{*} p<$ 0.05 vs OBX-veh, $\# p<0.05$ vs OBX-Flx.

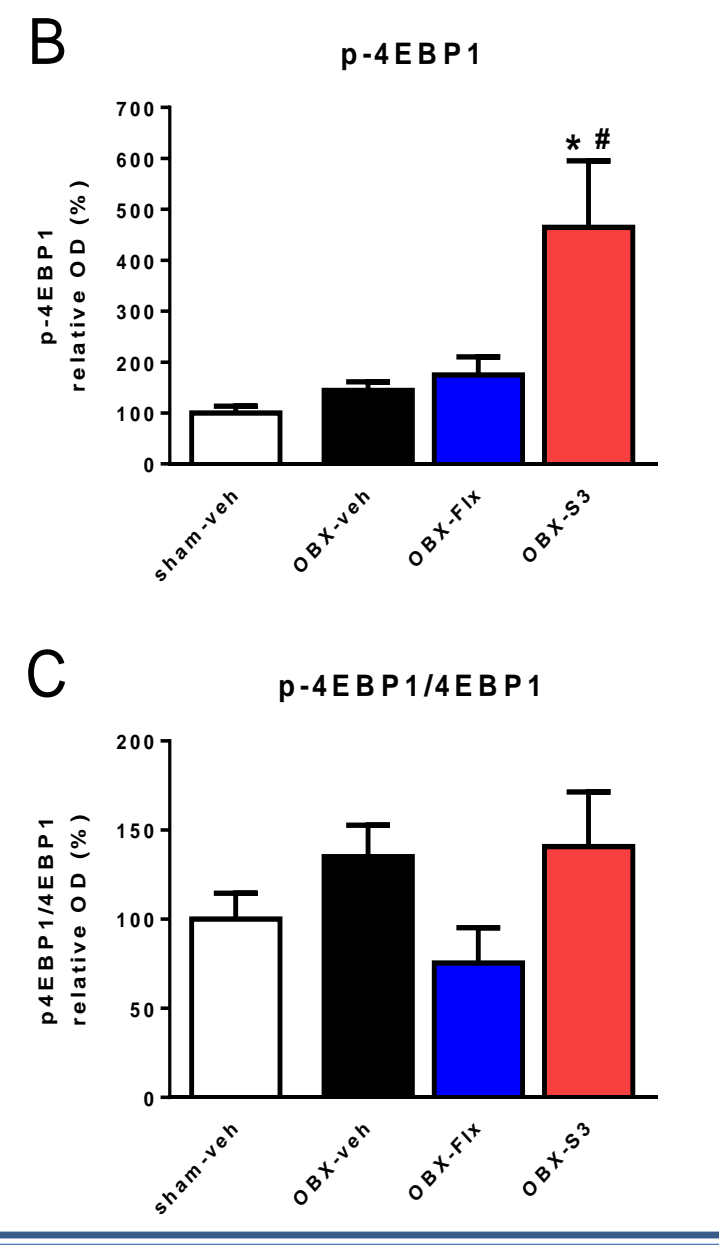

